European Association for the

Development of Renewable Energies,

Environment and Power Quality (EA4EPQ)
International Conference on Renewable Energies and Power Quality (ICREPQ'11)

Las Palmas de Gran Canaria (Spain), 13th to 15th April, 2011

\title{
Increasing transmission efficiency with advanced processing
}

\author{
I. Glesk, M. Nazri M. Warip and I. Andonovic \\ Department of Electronic and Electrical Engineering \\ University of Strathclyde \\ 204 George St, Glasgow G11XW, UK \\ Phone/Fax number:+44 141 5482529/+44 141 5524968, e-mail: ivan.glesk@eee.strath.ac.uk, \\ mohd.mohd-warip@eee.strath.ac.uk, 1.andonovic@eee.strath.ac.uk
}

\begin{abstract}
Not too long after its emergence, the Internet became an integral part of everyday lives of men in modern societies. It constantly fuels growing opportunities for new advanced services which can better respond to quickly changing society needs. Previously such services often led to increases in energy consumption which is today in a strong contrast with the global drive for a greener and energy more efficient environment. We need network infrastructures which will support these growing needs but at the same time will also stay zero-carbon emission complaint. Such requirements are more imperative today than were ever before. In optical IP networks the power consumption could be reduced via green photonics and concepts known as green networking [1]. In line with these concepts we proposed a novel energy savings approach based on so called "node hibernation" technique. This approach is designed to help reduce network power consumption and optimise its energy usage by taking advantage of a hibernation algorithm we have developed. Its implementation into the network management structure will save the energy, operating cost to network operators and will deliver reduced carbon footprint.
\end{abstract}

\section{Key words}

WDM, energy efficiency, GMPLS, green photonic networks, power consumption, carbon footprint.

\section{Introduction}

The Internet today offers an easy and convenient way to gain access to entertainment, social networking, and desired learning tools. The Internet became even the place to conduct business. These rapidly expanding activities result in power consumption increases which in turn could put our environment at a greater risk. However such risky trends could be hardly acceptable as a long term solution. Therefore novel greener concepts are gradually being developed and are being implemented into today's communication networks. Thanks to these innovative approaches with better energy conservation and decreased carbon emission we can have better environment and a healthier lifestyle. All such activities are considered green if they:

- generate or help to conserve energy;

- help to reduce greenhouse gas emission;

- will help to reduce pollution thus consequently lead to improvements in the public health.

By considering all the above we proposed an approach which could help to reduce power consumption and increase an energy efficiency in optical networks via optimisation and better power management of the control algorithm by implementing a novel approach to Generalized Multi-Protocol Label Switching (GMPLS) control plane. Implementation of this methodology results in a significant reduction of energy usage and helps to cut the network operating cost without compromising the connectivity/performance. We have also investigated the impact of our technique on the heat transfer rate in IP routers even further enhance energy savings. Our energy savings approach was studied on optical networks with Generalized Multi-Protocol Label Switching (GMPLS) protocol. Our performance evaluations of various power saving schemes were conducted successfully and included various challenging issue to help to make the current networking "greener" by reducing the overall power consumption thus lowering the carbon footprint.

\section{Network Energy Model}

In order to evaluate overall network power consumption and the consumed energy per one bit of transmitted data we use so called equivalent network energy model (see Fig. 1) which is based on the Internet Protocol/ Generalized Multi-Protocol Label Switching (IP/GMPLS) over optical layers. In our model, network carrier bandwidth of OC-192 and average energy consumption of $1019 \mathrm{~nJ}$ per bit was assumed after [2-4]. Symbol $G_{n}$ (see Fig. 1) denotes dissipated power of $10 \mathrm{~kW}$ within a router and consumption of $1000 \mathrm{~nJ} / \mathrm{bit}$. 
Symbol $Z_{\mathrm{n}} \mathrm{X}_{\mathrm{n}}$ represents Optical Cross-Connect (OXC) dissipating $100 \mathrm{~W}$ and consumes 10nJ/bit.

Symbol $W_{\mathrm{n}} \mathrm{W}_{\mathrm{n}}$ denotes Wavelength Division Multiplexing (WDM) part of the node with dissipating power of $120 \mathrm{~W}$ and energy consumption of $12 \mathrm{~nJ} / \mathrm{bit}$.

Symbol $A_{n}$ represents the consumption owing to ErbiumDoped Fibre Amplifiers (EDFAs) within connection spans placed at $70 \mathrm{~km}$ intervals and their power consumption is estimated to be $1 \mathrm{~W}$ with energy of $0.1 \mathrm{~nJ} / \mathrm{bit}$.

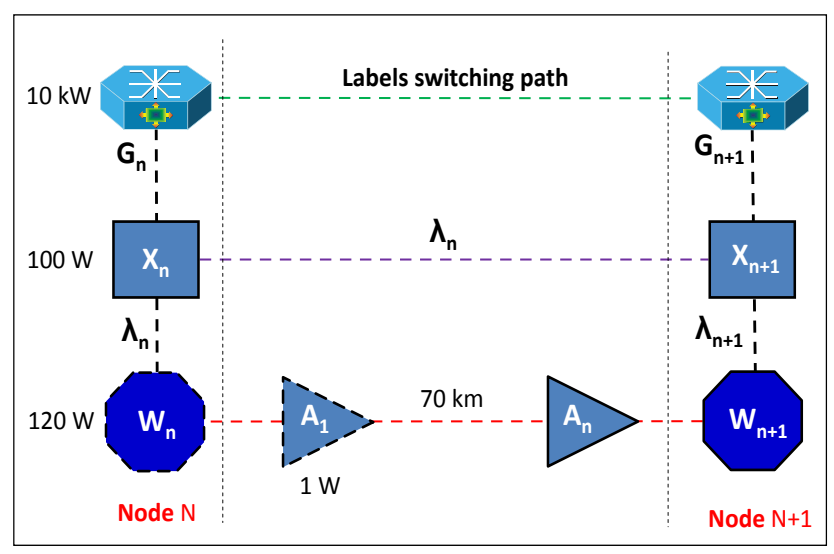

Fig. 1: Green Photonic Network Model.

Now, let us define the energy per bit consumed by the node as $\mathrm{E}_{\mathrm{b}}=\mathrm{P}_{\mathrm{T}} / \mathrm{c}$ where $\mathrm{P}_{\mathrm{T}}$ represents the node total power consumption and $\mathrm{c}$ is the bandwidth offered by the network link.

The total power consumption of the link is then defined as:

$$
P_{T}=\mathrm{P}_{\mathrm{g}}+\mathrm{P}_{\mathrm{x}}+\mathrm{P}_{\mathrm{a}}+\mathrm{P}_{\mathrm{w}}+\mathrm{P}_{\mathrm{tr}}
$$

where $\mathbb{P}_{g} \mathbb{P}_{\mathbb{W}^{x}} \mathbb{P}_{\mathbb{Q}^{v}} \mathbb{P}_{\mathbb{W}} \mathbb{P}_{\mathrm{ty}} \mathrm{P}_{\mathrm{g}}, \mathrm{P}_{\mathrm{x}}, \mathrm{P}_{\mathrm{a}}, \mathrm{P}_{\mathrm{w}}$, and $\mathrm{P}_{\mathrm{tr}}$ represent power consumed by the IP/GMPLS router, OXC, EDFA, WDM and transmitter, respectively.

The total energy $E_{b i t}$ per one data bit which is needed to support the network offered load is then defined as:

$$
E_{b i t}=\left[\Sigma\left(\mathrm{P}_{\mathrm{g}}+\mathrm{P}_{\mathrm{w}}+\mathrm{P}_{\mathrm{tr}}\right) / \mathrm{C}\right]+\left[\sum \varepsilon_{\mathrm{a}}+(\rho+1) \varepsilon_{\mathrm{x}}\right]+\delta
$$

where $\varepsilon_{\mathrm{a}}$ is energy consumed by EDFA; $\varepsilon_{\mathrm{x}}$ is the energy consumed by the node's OXC; $\delta$ represents the noise factor associated with the Bit Error Rate (BER) and a heat transfer rate in network equipments; and finally, $\rho$ is number of hops.

\section{Improving Network Energy Efficiency}

As already motioned our energy saving model was investigated on models with IP/GMPLS control plane. The IP/GMPLS control plane can be configured to perform a power aware routing including the network data traffic control [2]-[3].

Figure 2 depicts the concept of the IP/GMPLS in a Wavelength Route Network (WRN). It schematically shows an IP/GMPLS control plane, Optical Cross-
Connect (OXC), Erbium Doped Fibre Amplifiers (EDFAs) for signal in line amplification, transmitter Tx, receiver $\mathrm{Rx}$, and a 40 channels-based Wavelength Division Multiplexing (WDM) unit operating at OC-192 ( 10 Gigabit per second), respectively.

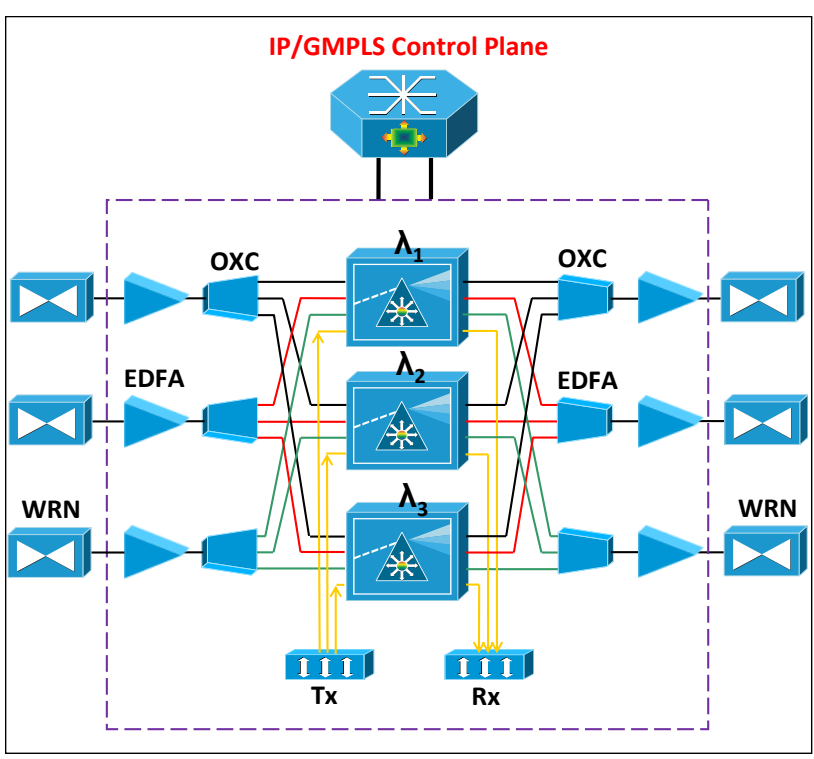

Fig. 2. Concept of IP/GMPLS over optical network.

Figure 3 gives more detailed diagram of the implementation of the $\mathrm{OXC}$ and the Router, it is also showing Resource Reservation Protocol with Traffic Engineering (RSVP-TE) unit which is responsible for signalling, Open Shortest Path First with Traffic Engineering (OSPF-TE) unit for handling routing function, Network Controller, OXC with wavelength route-table, Router unit, and the Energy monitoring or 'Green Unit' (GU).

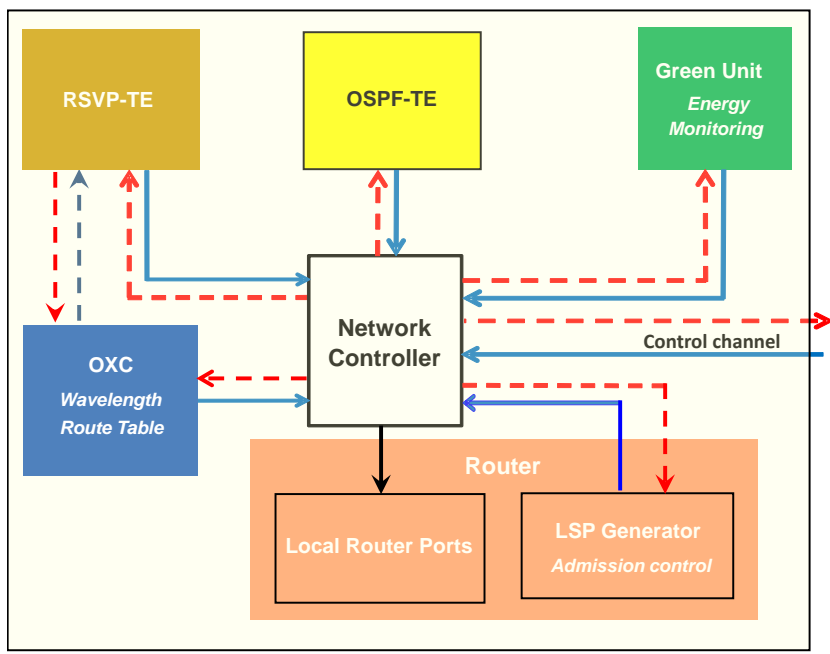

Fig. 3. OXC and Router - block diagram.

The control plane follows the GMPLS standards based on a single common control channel it performs local resource management, routing and signalling, calls set-up and connection control. Green Unit records the overall power consumption of the entire Node. GU also communicates with the Network Controller through the control channel and provides node's energy status and controls its energy consumption. Based on this 
information the Network Controller can decide which "Hibernation mode" option would be optimal to invoke in order to maintain desired network performance and at the same time to deliver the most energy savings.

\section{Concept of Proposed Hibernation Model}

Let us assume that IP/GMPLS nodes within studied optical network are linked by bidirectional pairs of single mode fibres, that each message length is fixed at 256 bytes, that all links use the equal number of wavelengths (eight), and the nodal processing delay is $20 \mathrm{~ms}$; any other network parameters we used in our simulations were based on [4]-[7].

Let us now formally define the "Hibernation mode" $H^{m}$. We assume IP/GMPLS nodes within the optical network:

$$
H^{m} \in \sum_{\forall p q r}\left[\Psi_{p} \cup \Phi_{q} \cup \Omega_{r}\right]
$$

where $m$ represents the particular configuration of the "hibernation mode" within nodes grouping $\Psi_{\mathrm{p}}$, links segmentations $\Phi_{\mathrm{q}}$, and wavelengths partitionings $\Omega_{\mathrm{r}}$. Parameters $\Psi_{\mathrm{p}}, \Phi_{\mathrm{q}}, \Omega_{\mathrm{r}}$ represent individual groups of network nodes $\Psi_{\mathrm{p}}=\left\{\Psi_{1}, \Psi_{2}, \Psi_{3}, . ., \Psi_{\mathrm{p}}\right\}$, segmentation of network links $\Phi_{\mathrm{q}}=\left\{\Phi_{1}, \Phi_{2}, \Phi_{3}, . ., \Phi_{\mathrm{q}}\right\}$, and wavelengths partitionings $\Omega_{\mathrm{r}}=\left\{\Omega_{1}, \Omega_{2}, \Omega_{3}, . ., \Omega_{\mathrm{r}}\right\}$, respectively. Integer numbers p, q, r, u, v, w represent number of groups, segmentations, partitions, nodes, links, and wavelengths, respectively and range from 1 to the total number of node groups, segmentations or partitionings. (Rem.: the number of nodes depends on the network size whilst the number of segmentations depends on the number of connections within the network and the number of partitions is determined by the number of wavelengths $\lambda_{w}$ within network links.

Based on the above concept of "Hibernation mode" the network power consumption and network performance was studied by implementing following three steps:

First a Grouping $\left(\mathrm{H}^{\Psi}\right)$ was performed - network nodes were sorted into different groups based on their proximity (geographical grouping).

Second, identified idled links were shut down to invoke additional energy conservation by a process we call Links Segmentation $\left(\mathrm{H}^{\Phi}\right)$.

Third a Wavelengths Partitioning $\left(\mathrm{H}^{\Omega}\right)$ was implemented to further optimise energy consumption by tearing down all idled lightpaths within the node link(s) and also by sub grouping then based on their activity status

\section{Obtained Results and Discussion}

We used a discrete event modelling tool known as OMNet++ (Object Modular Network Tested in $\mathrm{C}++)$ to evaluate the effectives of our "Node hibernation" technique. The technique was applied under different $G_{i}$ grouping scenarios (discussed later) to selected nodes/groups/segments within the studied network. The network topology used in our simulation was the National Science Foundation (NSFnet) network schematically depicted in Fig. 4. [4], [7]. It has a bandwidth of OC-192 and bidirectional single mode fibre links are installed between nodes.

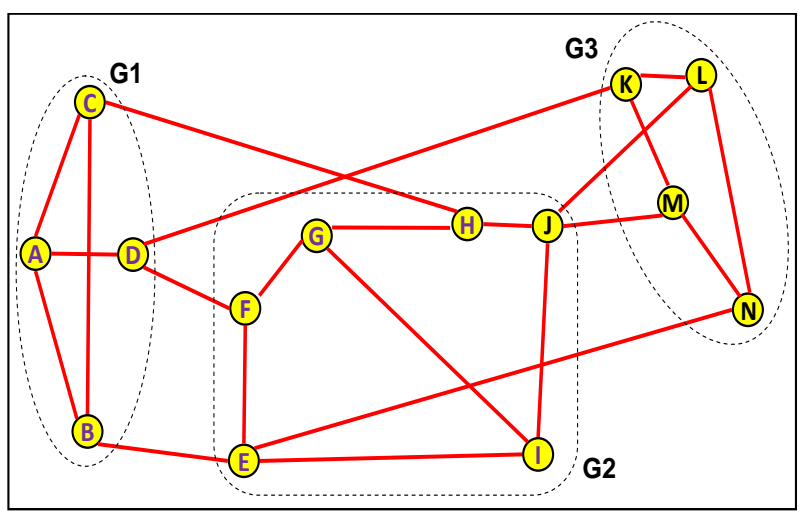

Fig. 4. National Science Foundation (NSFnet) network.

The NSFnet network has a Partial Network Topology with 14 nodes and 22 bidirectional fibre links [4], [7]. We also assume that the NSFnet has uniformly distributed traffic. Used EDFA amplifiers bear minimum power consumption of $1 \mathrm{~W}$ and are placed at every $70 \mathrm{~km}$. The standard GMPLS signalling and routing protocol follows the Internet Engineering Task Force (IETF) standard [8][9]. The performance metrics included in our simulations take into account the average power consumption, blocking probability and average request blocking [2], [5], [7], [10]-[11]. The blocking probability $P_{b}$ is determined by using Erlang-B formula [12]-[15] as function of offered load and the number of wavelengths. The total number of connection requests that are available is the offered load (traffic load) denoted in Erlangs. The arrival connection requests are independent Poisson processes with an $\alpha$ arrival rate and the queue lengths exponentially distributed with an expected service rate time of $1 / \mu$ measured in seconds.

The earlier mentioned node groupings $G_{i}$ were based on the membership called Geographical or Localized (see Fig. 4). See nodes listing belonging to different groups $G_{i}$ in the Table I.

Table I. - Nodes Grouping Categorization.

\begin{tabular}{|c|c|}
\hline Group $\mathrm{G}_{\mathrm{i}}$ & Selected nodes in the group \\
\hline $\mathrm{G} 1$ & $\{\mathrm{~A}, \mathrm{~B}, \mathrm{C}, \mathrm{D}\}$ \\
\hline $\mathrm{G} 2$ & $\{\mathrm{E}, \mathrm{F}, \mathrm{G}, \mathrm{H}, \mathrm{I}\}$ \\
\hline $\mathrm{G} 3$ & $\{\mathrm{~J}, \mathrm{~K}, \mathrm{~L}, \mathrm{M}, \mathrm{N}\}$ \\
\hline
\end{tabular}

In our simulations we considered hibernation settings applied to the following groups of nodes:

- All " $\mathrm{G}_{\mathrm{i}}$ " Groups are $\mathrm{ON}$;

- All " $\mathrm{G}_{\mathrm{i}}$ ” Groups are OFF;

- Groups “G1 \& G2” are OFF; 
- Groups “G2 \& G3” are OFF;

- Group "G1" is OFF;

- Group "G2" is OFF;

- Group "G3" is OFF.

We investigated groups and segments configurations allowing the hibernation to be applied to designated nodes and links. (Remark: Hibernation mode is activated when groups and segments are set to off state e.g., nodes disable their unused ports/interfaces, Mux/deMux capabilities, signaling gates, unused wavelengths, etc., and keep only the minimum required network activities. Such conditions could be noticed by observing whether nodes and links are in their idle states or whether there is any active traffic in the given part of the network.

Figure 5 illustrates the difference in the average power consumption for various "Geographical Nodes grouping" versus the offered network load (see the legend in Fig. 5).

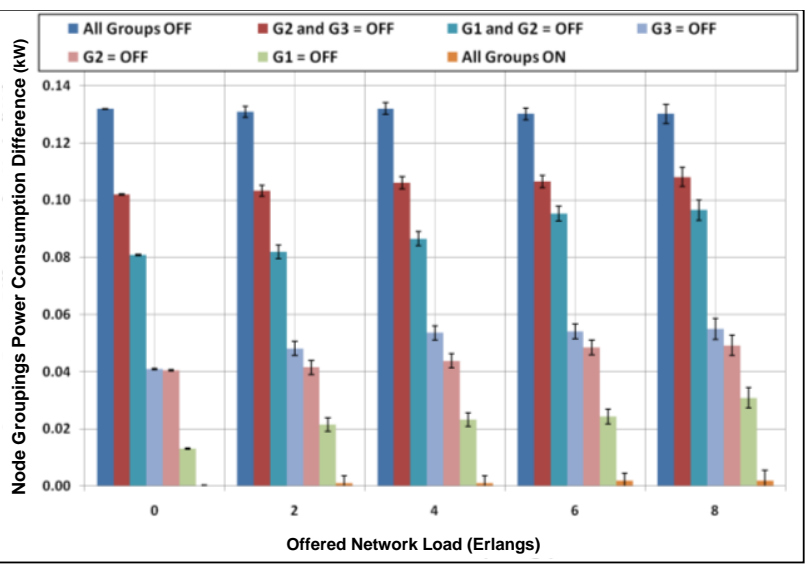

Fig. 5. Simulation results for different Geographical Nodes groupings.

By comparing obtained results shown in Fig. 5 and Fig. 6

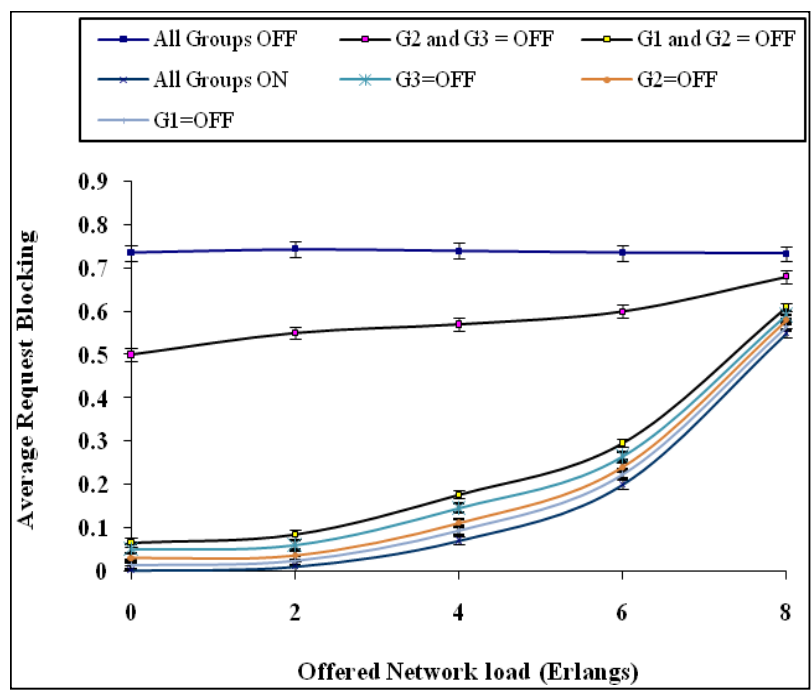

Fig. 6. Request blocking for Geographical nodes groupings.

we observe that in the case of All " $\mathrm{G}_{\mathrm{i}}$ "'Groups are OFF (case when the node's unused functionalities are deactivated), about $0.135 \mathrm{~kW}$ of energy can be saved (see
Fig. 5) but the probability of blocking is $\sim 70 \%$ (see Fig. 6 ) which will result in a congested connection.

If Groups "G2 \& G3" are OFF or Groups "G1" \& "G2" are OFF, the energy savings of $0.10 \mathrm{~kW}$ or $0.90 \mathrm{~kW}$, respectively is delivered (see Fig. 5). However, the corresponding blocking probability would be $50 \%$ or $10 \%$, respectively (see Fig. 6).

Based on our simulations we can also conclude that the NSFnet network will become very inefficient and congested for network loads exceeding 8 Erlangs.

From theses presented results can be also predicted that with the energy consumption decreases the probability of blocking increases which is either due to a lesser wavelength availability or because the node(s) are in their "Hibernation mode" state as the result of power savings activities imposed via network management.

In conclusion, this paper presents the novel approach to energy savings in IP/GMPLS based optical networks. We examined the impact of the "Hibernation mode" approach on the performance of the optical IP network while simultaneously looking on energy savings of selected nodes groupings. The selection was done on their Geographical location/position inside the NSFnet network. Our results showed that the applied Hibernation mode approach can lead to energy savings in IP/optical networks.

\section{Acknowledgement}

Authors would like to thank to GRPe partnership for financially supporting this research. Mr. M. Nazri M. Warip would like to thank Ministry of Higher Education of Malaysia and the University Malaysia Perlis, Kangar, Malaysia for providing his $\mathrm{PhD}$ scholarship.

\section{References}

[1] M.S. Lebby, Green Photonics Technology and Markets, IET Electronic Letters, Vol. 45. pp. 1007-1007 (2009).

[2] S. Albarrak, "Failure recovery in distributed GMPLSbased ip-over-optical networks," PhD Thesis, University of Strathclyde, UK (2008).

[3] J. Balinga, R. Ayre, K. Hinton, W.V. Sorin and R.S. Tucker, Energy Consumption in Optical IP Networks, IEEE Journal of Lightwave Technology, Vol. 27. pp. 2391-2403 (2009).

[4] B.G. Bathula, M. Alresheedi and J.M.H. Elmirghani, Energy Efficient Architectures for Optical Networks, in Proc. London Communications Symposium (LCS), London, UK, (2009).

[5] Cisco System Data Sheets, [Online]. Available: http://www.cisco.com.

[6] MRV Communications Inc Data Sheet, [Online]. Available: http://www.mrv.com.

[7] G. Shen and R.S. Tucker, Energy-Minimized Design for IP Over WDM Networks, IEEE Journal of Optical Communication Network, vol.1. no.1, pp.176-186 (2009).

[8] L. Berger, Generalized Multi-Protocol Label Switching (GMPLS) Signalling Functional Description, [Online]. Available: http://www.faqs.org/ftp/rfc/pdf/rfc3471.txt.pdf, (2003). 
[9] J. Lang, Generalized Multi-Protocol Label Switching (GMPLS) Signalling Resource Reservation ProtocolTraffic Engineering (RSVP-TE) Extension, IETF RFC 3473 [Online]. Available at: http://www.faqs.org/rfcs/rfc3473.html.

[10] J. Chabarek, J. Sommers, P. Barford, C. Estan, D. Tsiang and S. Wright, Power Awareness in Network Design and Routing, in IEEE Proc. $27^{\text {th }}$ Conference on Computer Communications INFOCOM 2008, Phoenix, USA, pp. 1130-1138 (2008).

[11] N. Vasic, P. Bhurat and D. Kostic, Energy-Aware Traffic Engineering, EPFL Technical Report NSL-REPORT2008-004, Switzerland (2008).

[12] R. Barry, "Wavelength Routing for All-Optical Networks", PhD Dissertation, Department of Electrical Engineering and Computer Science, MIT, 1993.

[13] R.A. Barry and P.A. Humblet, "Models of Blocking probability in all-optical networks with and without wavelength changers", IEEE Journal on Selected Areas in Communications, Vol. 14. No. 5. pp. 858-867 (1996).

[14] A. Sridharan and K.N. Sivarajan, "Blocking in All-Optical Networks", IEEE/ACM Transactions on Networking, Vol. 12. No. 12, pp. 364-397 (2004).

[15] R. Ramaswami, K.N. Sivarajan and G.H. Sasaki, "Optical Networks: A Practical Perspective", $3^{\text {rd }}$ Ed., MorganKauffmann Publishers (2010). 